\title{
INTERAÇÃO GENÓTIPOX AMBIENTE SOBRE CARACTERÍSTICAS PRODUTIVAS E REPRODUTIVAS DE FÊMEAS NELORE
}

\author{
GENOTYPE BY ENVIRONMENT INTERACTION ON PRODUCTIVE AND REPRODUCTIVE \\ TRAITS OF FEMALES OF THE NELLORE BREED
}

\begin{abstract}
Silveira, M.V. ${ }^{1 *}$; Souza, J.C. ${ }^{1 A}$; Silva, L.O.C. ${ }^{2 A}$; Freitas, J.A. ${ }^{3}$; Gondo, A. ${ }^{2 B}$ e Ferraz Filho, P.B. ${ }^{1 B}$
${ }^{1}$ Programa de pós-graduação em Ciência Animal. Universidade Federal de Mato Grosso do Sul. Campo Grande, MS. Brasil. *mauriciozootecnia@gmail.com; AJulio.souza@ufms.br; Bpaulo.ferraz@ufms.br ${ }^{2}$ Empresa Brasileira de Pesquisa Agropecuária. Embrapa Gado de Corte. Campo Grande, MS. Brasil. Alocs@cnpgc.embrapa.br; Bandrea@cnpgc.embrapa.br

3Universidade Federal do Paraná. UFPR. Palotina, PR. Brasil. freitasjaf@ufpr.br
\end{abstract}

\section{AdDitionAL KEYWORDS}

Beef cattle. Genetic parameters. Heritability.

\section{RESUMO}

Foram estimados parâmetros genéticos e avaliada a interação genótipo $x$ ambiente para peso ajustado aos 420 dias de idade, idade ao primeiro parto e intervalo entre o primeiro e segundo parto, em fêmeas da raça Nelore criadas em três regiões de produção do estado de Mato Grosso do Sul. O modelo estatístico continha os efeitos genéticos aditivo direto e residual (efeitos aleatórios) e, como fixos, os efeitos de fazenda e do grupo de contemporâneos. As análises foram executadas sob modelo animal do tipo uni e bicaracterística. As estimativas de herdabilidade foram 0,$41 ; 0,16$ e 0,09 para P420, IPP e IEP12, respectivamente. Ao se analisar usando a correlação de Spearman para avaliar se houve variação na ordenação da diferença esperada na progênie, constatou-se alteração na classificação dos reprodutores de região para região. Estes resultados evidenciam a existência de interação genótipo $\mathrm{x}$ ambiente para as características avaliadas, havendo possibilidade de os reprodutores selecionados como de mérito genético superior para uma região não o serem para outras.

\section{SUMMARY}

Genetic parameters were estimated and the genotype x environment interaction was evaluated for weight adjusted for 420 days old, age at first

\section{PalaVRas CHAVE ADICIONAIS}

Gado de corte. Herdabilidade. Parâmetros genéticos.

calving and first calving interval, in females of the Nelore raised in three regions in the state of Mato Grosso do Sul. The statistical model contained the genetic effects and direct additive (random effects) and residual, as fixed, the effects of farm and contemporary group. Analyses were performed with univariate and bivariate animal model. Heritability estimates were $0.41 ; 0.16 ; 0.09$; for $\mathrm{W} 420, \mathrm{AFC}$ and $\mathrm{FCl}$, respectively. When was utilized the Spearman correlation to compare the variation in the ordering of the expected progeny differences, there was noted the change in classification of the sires from region to region. These results show evidence of genotype by environment interaction for the traits studied, with possibility of breeders selected as top genetic merit for a region not be for others.

\section{INTRODUÇÃO}

Entre as características determinantes da eficiência reprodutiva dos rebanhos bovinos de corte, destacam-se a idade à primeira parição e o intervalo de parto, relacionado à taxa de natalidade e à longevidade produtiva das vacas. Quanto mais jovem a novilha tenha o primeiro parto, mais rápido o retorno do investimento feito pelo pecuarista na criação e manutenção desse 


\section{SILVEIRA, SOUZA, SILVA, FREITAS, GONDO E FERRAZ FILHO}

animal até a idade reprodutiva. Por sua vez, intervalos de partos menores resultarão em maior retorno sobre os custos fixos e operacionais envolvidos no rebanho de cria, pois o aumento da taxa de natalidade reflete em aumento de receita (Perotto et al., 2006). Animais superiores em uma região ou país podem não ter o mesmo resultado em outro. Isso sugere que a escolha de um animal apropriado para uma determinada região (ou país) é específico, podendo este não ter a mesma resposta em outra região (Souza et al., 2003; Toral et al., 2004; Payá et al., 2007; Lopes et al., 2008; Espasandin et al., 2011). O objetivo foi investigar a existência de interação genótipo $\mathrm{x}$ ambiente para as características idade ao primeiro parto, intervalo entre o primeiro e segundo parto e peso ajustado aos 420 dias de idade em fêmeas da raça Nelore, bem como determinar a estimativa dos parâmetros genéticos destas.

\section{MATERIALEMÉTODOS}

Informações de 30461 fêmeas da raça Nelore, nascidas entre os anos de 1978 e 2008 criadas a pasto, nas regiões do Pantanal Sul (R1), Alto Taquari-Bolsão (R2) e Campo Grande-Dourados (R3), pertencencentes ao Arquivo Zootécnico Nacional raças zebuínas (ABCZ/Embrapa). O número de animais avaliados, reprodutores, fazendas, grupos de contemporâneos e a média observada de cada característica por região (Arruda e Sugai, 1994) encontram-se na tabela I. As características estudadas foram peso ajustado para os 420 dias de idade (P420), idade ao primeiro parto (IPP) e in-tervalo entre o primeiro e segundo parto (IEP12). Os componentes de (co) variância e parâmetros genéticos foram estimados utilizando-se o método REML, programa MTDFREML (Boldman et al., 1995), com modelo animal completo.

Para avaliar a interação, considerou a mesma característica como característica diferentente em duas regiões (Souza et al., 2003). Valor da correlação genética menor que 0,80 , considerou-se que a interação genótipo $\mathrm{x}$ ambiente foi significativa $(\mathrm{p}<0,05)$. O efeito da interação genótipo $x$ ambiente foi avaliado também por meio da mudança na ordem de classificação dos reprodutores com base na DEP (diferença esperada na progênie) das características (P420, IPP e IEP12) avaliadas entre as regiões aos pares e consideradas como sendo características diferentes. A similaridade entre a classificação dos reprodutores entre as regiões foi avaliada por meio da correlação de Spearman.

\section{RESULTADOSEDISCUSSÃO}

Os valores de $\sigma_{\mathrm{a}}^{2}, \sigma^{2}, \mathrm{~h}^{2} \pm \mathrm{EP}, \mathrm{e}^{2} \pm \mathrm{EP}$, para a característica $\mathrm{P} 420$, foram os seguintes: $453,35,1100,05,0,41 \pm 0,02$, $0,59 \pm 0,02$, respectivamente; para a característica IPP obteve-se os seguintes valores: 3365,$85 ; 21036,56 ; 0,16 \pm 0,01 ; 0,84 \pm 0,01$, respectivamente; e para a característica IEP12 foram obtidos os seguintes valores: 2135,$87 ; 23731,89 ; 0,09 \pm 0,02 ; 0,91 \pm 0,02$, respectivamente. Valores semelhantes foram encontrados por Silveira et al., (2004); Toral et al., (2004); Lopes et al., (2008). As estimativas de $\mathrm{h}^{2}$ obtidas para a característica $\mathrm{P} 420$ para a região $\mathrm{R} 1, \mathrm{~h}^{2}=0,47$; para a região $\mathrm{R} 2, \mathrm{~h}^{2}=0,37$; e para a região $\mathrm{R} 3, \mathrm{~h}^{2}=0,44$. As correlações genéticas entre as regiões $\mathrm{R} 1 \mathrm{e}$ R2: 0,80; entre as regiões R1 e R3: 0,62; e entre as regiões R2 e R3: 0,70, portanto, constatou-se o efeito da interação genótipo $\mathrm{x}$ ambiente entre as regiões $\mathrm{R} 1$ e R3 e entre $\mathrm{R} 2$ e R3. A estimativa de $\mathrm{h}^{2}$ para a característica IPP na região $\mathrm{R} 1$ foi 0,25 ; e nas regiões R2 e R3 foram 0,17; para essa mesma característica a correlação genética entre as regiões R1 e R2 foi 0,62; entre R1 e R3 foi 0,72; e entre as regiões R2 e R3 foi 0,51. Para a característica IEP12, a estimativa de $h^{2}$ para região $\mathrm{R} 1$ foi 0,08 ; para a região $\mathrm{R} 2$ foi 0,06 ; e para a região R3 foi 0,11. A correlação genética entre as regiões R1 e R2 foi 1,00; entre as regiões R1 e R3 foi 0,89 ; e entre as regiões $\mathrm{R} 2$ e R3 foi 0,76 . 


\section{INTERAÇÃO GENÓTIPOXAMBIENTE SOBRE CARACTERÍSTICAS DE FÊMEAS NELORE}

A correlação de Sperman estimada para a característica $\mathrm{P} 420$ entre as regiões $\mathrm{R} 1$ e R2 foi 0,94 (com 239 reprodutores em comum); entre as regiões R1 e R3 foi 0,83 (com 349 reprodutores em comum); e entre as regiões R2 e R3 foi 0,90 (com 627 reprodutores em comum). Para a característica IPP a correlação de Sperman entre as regiões R1 e R2 foi 0,82 (com 214 reprodutores em comum); entre as regiões R1 e R3 foi 0,91 (com 316 reprodutores em comum); e entre as regiões R2 e R3 foi 0,75 (com 591 reprodutores em comum). Para a característica IEP12, a correlação de Spearman entre as regiões R1 e R2 foi 1,00 (com 167 reprodutores em comum); entre as regiões R1 e R3 foi 0,99 (com 240 reprodutores em comum); e entre as regiões R2 e R3 foi 0,96 (com 468 re-

Tabela I. Quantidade de animais (N), reprodutores (REP), fazendas (FAZ), grupos de contemporâneo $(G C)$, média e desviopadrão (DP) de acordo com as características estudadas. (Number of animals (N), Sires (REP), farms (FAZ), contemporany groups, mean and standard deviation (DP) according to studied traits).

\begin{tabular}{lrcccc}
\hline Região & $N$ & REP & FAZ & GC & Média $\pm D P$ \\
\hline P420 $(\mathrm{kg})$ & & & & & \\
R1 & 2395 & 474 & 73 & 67 & $237,3 \pm 40,12$ \\
R2 & 9719 & 1352 & 285 & 67 & $225,6 \pm 38,37$ \\
R3 & 18347 & 1204 & 527 & 68 & $241,0 \pm 41,10$ \\
Total & 30461 & 3030 & 885 & 68 & $235,8 \pm 40,78$ \\
IPP (dias) & & & & \\
R1 & 2144 & 454 & 68 & 67 & $1195,5 \pm 166,02$ \\
R2 & 9386 & 1327 & 277 & 67 & $1211,4 \pm 163,53$ \\
R3 & 17979 & 2149 & 513 & 67 & $1177,8 \pm 163,71$ \\
Total & 29509 & 3930 & 858 & 67 & $1189,8 \pm 164,55$ \\
IEP12 (dias) & & & & \\
R1 & 1448 & 356 & 56 & 65 & $566,2 \pm 178,72$ \\
R2 & 6524 & 1080 & 260 & 64 & $543,4 \pm 156,22$ \\
R3 & 11924 & 1739 & 462 & 65 & $534,1 \pm 163,52$ \\
Total & 19896 & 3175 & 778 & 65 & $539,5 \pm 162,56$ \\
\hline
\end{tabular}

R1: Pantanal Sul; R2: Alto Taquari, Bolsão; R3: Campo Grande, Dourados. produtores em comum). Ambas estimativas de $h^{2}$ para as características reprodutivas foram de magnitude baixa indicando que a maior causa de variação que atuante sobre elas pode ser devida a fatores ambientais. A $\mathrm{h}^{2}$ para IPP foi semelhante ao obtido por Dias et al. (2004). Porém, inferior aos valores encontrados por Bertazzo et al. (2004) e Gressler et al. (2005), os quais obtiveram os valores de 0,36 e 0,27 , respectivamente.

Os valores $h^{2}$ para a característica IEP12 também foi semelhante aos encontrados nas fontes de literatura, que variaram de 0,01 a 0,10 (Bertazzo et al., 2004; Silveira et al., 2004; Gressler et al., 2005; Carolino et al., 2007). As estimativas de $\mathrm{h}^{2}$ quando as regiões (R1, R2 e R3) foram avaliadas separadamente e sofrem alterações quando comparadas as análises que utilizam o arquivo geral de dados. Os valores demonstram que quando as informações de uma região homogênea são avaliadas separadamente ocorre alteração nas estimativas de $\mathrm{h}^{2}$.

Com base nas estimativas de correlação genética, observou-se que para a característica P420 ocorreu interação entre as regiões R1 e R3 e, entre R2 e R3; para IPP ocorreu interação entre as três regiões; para IEP12 ocorre interação entre R2 e R3. Trabalhando com peso ao desmame em animais da raça Nelore, e utilizando a mesma metodologia, Souza et al. (2003) também detectaram a existência de interação genótipo $\mathrm{x}$ ambiente entre as regiões do Alto Taquari, Bolsão (R2) e Campo Grande, Dourados (R3). Carolino et al. (2007) trabalhando com características reprodutivas (IPP e IEP) em bovinos da raça Alentejana não observaram indícios de interação genótipo x ambiente.

A correlação de Spearman com base na DEP de touros que possuem filhas nas regiões analisadas aos pares, foi outro indicativo da existência da interação genótipo x ambiente. Esta correlação indica qual a magnitude da combinação na classificação com base nas DEP's de reprodutores ava- 


\section{SILVEIRA, SOUZA, SILVA, FREITAS, GONDO E FERRAZFILHO}

liados em duas regiões diferenciam-se ou assemelham-se entre si. Sendo que entre as regiões (R1 e R2 para P420 e IEP12; R1 e R3 para IEP12) que não se detectou interação genótipo x ambiente por meio de correlações genéticas, a correlação de Spearman situouse mais próximo de 1,00 (acima de 0,93).

Os animais identificados como melhoradores em um determinado ambiente não serão, necessariamente, os melhores quando

\section{BIBLIOGRAFIA}

Arruda, Z.J.e Sugai, Y. 1994. Regionalização da pecuária bovina no Brasil. Editora: EMBRAPACNPGC. Campo Grande, MS. 144 pp.

Bertazzo, R.P.; Freitas, R.T.F.; Gonçalves, T.M.; Pereira, I.G.; Eler, J.P.; Ferraz, J.B.S.; Oliveira, A.I.G. e Andrade, I.F. 2004. Parâmetros genéticos de longevidade e produtividade de fêmeas da raça Nelore. Rev Bras Zootecn, 33: 1118-1127.

Boldman, K.G.; Kriese, L.A.; Van Vleck, L.D.; Van Tassell, C.P. and Kachman, S.D. 1995. A manual for use of MTDFREML. A set of programs to obtain estimates of variances and covariances [DRAFT]. <ftp://aipl.arsusda.gov/pub/outgoing/ mtdfreml/mtdfrman.pdf> (15/09/2013).

Carolino, N.; Gama, L.T. e Espadinha, P. 2007. Interações genótipo $x$ ambiente em caracteres reprodutivos e de crescimento de bovinos Alentejanos. Arch Zootec, 56: 634-640.

Dias, L.T.; El Faro, L. e Albuquerque, L.G. 2004. Estimativas de herdabilidade para idade ao primeiro parto de novilhas da raça Nelore. Rev Bras Zootecn, 33: 97-102.

Espasandin, A.C.; Urioste, J.I.; Campos, L.T. and Alencar, M.M. 2011. Genotype × country interaction for weaning weight in the Angus populations of Brazil and Uruguay. Rev Bras Zootecn, 40: 568-574.

Gressler, M.G.M.; Pereira, J.C.C.; Bergmann, J.A.G.; Andrade, V.J.; Paulino, M.F. e Gressler, S.L. 2005. Aspectos genéticos do peso à desmama e de algumas características reprodutivas de fêmeas Nelore. Arq Bras Med Vet Zoo, 57: 533-538.

Lopes, J.S.; Rorato, P.R.N.; Weber, T.; Boligon, A.A.; Comin, J.G. e Dornelles, M.A. 2008. Efeito transferidos para outro ambiente.

\section{CONCLUSÕES}

A classificação dos reprodutores com base no desempenho de suas progênies variou de uma região para outra. Dessa forma, a escolha correta dos reprodutores para fazendas específicas é fundamental para maximização do progresso genético.

da interação genótipo $x$ ambiente sobre o peso ao nascimento, aos 205 e aos 550 dias de idade de bovinos da raça Nelore na Região Sul do Brasil. Rev Bras Zootecn, 37: 54-60.

Payá, S.B.; Simões, A.C.; Ferraz Filho, P.B.; Silva, L.O.C. e Souza, J.C. 2007. Causas de variações não genéticas e interações estação x região em pesos de animais de rebanhos Nelore Mocho em áreas inclusas na Região Pecuária de Leiteiras. Arch Vet Sci, 12: 8-12.

Perotto, D.; Miyagi, A.P.; Souza, J.C.; Moletta, J.L. e Freitas, J.A. 2006. Estudos de características reprodutivas de animais da raça Canchim, criados a pasto, no estado do Paraná, Brasil. Arch Vet Sci, 11: 1-6.

Silveira, J.C.; McManus, C.; Mascioli, A.S.; Silva, L.O.C; Silveira, A.C.; Garcia, J.A.S e Louvandini, H. 2004. Fatores ambientais e parâmetros genéticos para características produtivas e reprodutivas em um rebanho Nelore no estado do Mato Grosso do Sul. Rev Bras Zootecn, 33: 1432-1444.

Souza, J.C.; Gadini, C.H.; Silva, L.O.C.; Ramos, A.A.; Euclides Filho, K.; Alencar, M.M.; Ferraz Filho, P.B. and Van Vleck, L.D. 2003. Estimates of genetic parameters and evaluation of genotype $x$ environment interaction for weaning weight in Nellore cattle. Arch Latinoam Prod Anim, 11: 94-100.

Toral, F.L.B. ; Silva, L.O.C.; Martins, E.N.; Gondo, A. e Simonelli, S.M. 2004. Interação genótipo $x$ ambiente em características de crescimento de bovinos da raça Nelore no Mato Grosso do Sul. Rev Bras Zootecn, 33: 1445-1455.

Archivos de zootecnia vol. 63, núm. 241, p. 226. 